\title{
Correlation between the Occlusion Site and Clinical Outcome after Acute Ischemic Stroke
}

\author{
Kentaro Suzuki ${ }^{1}$, Junya Aoki ${ }^{1}$, Takao Kanzawa ${ }^{2}$, Yasuhiro Nishiyama ${ }^{1}$, Yohei Takayama ${ }^{1}$, \\ Hiroaki Kimura ${ }^{3}$, Satoshi Takahashi ${ }^{4}$, Tadashige Kano ${ }^{4}$, Kazunori Akaji ${ }^{4}$, Yoshio Tanizaki ${ }^{4}$, \\ Kazumi Kimura $^{1}$ and Ban Mihara ${ }^{3}$
}

\begin{abstract}
Objective The significance of early mild clinical improvement after intravenous tissue plasminogen activator (IVtPA) treatment is unclear. Therefore, we examined whether the timing of clinical improvement after IVtPA predicted the clinical outcome at 3 months.

Methods Consecutive patients with acute cerebral infarction in the anterior circulation who received IVtPA treatment within 3 hours of the onset of symptoms were enrolled in the study. Patients were classified according to the timing of clinical improvement [early responder (ER), National Institutes of Health Stroke Scale (NIHSS) score improved $\geq 4$ points or who had a score of 0 within 2 hours after IVtPA; late responder (LR), a similar improvement between 2 and 24 hours; and non-responder (NR)] and according to the arterial occlusion site (P group, internal carotid artery and proximal middle cerebral artery M1 region; and D group, distal M1 and M2).

Results Ninety-three patients [median age, 74 (67-79) years; 54 men (58\%); median NIHSS score, 11 (716)] were enrolled in the study. The $P$ group consisted of $48(52 \%)$ patients and the $D$ group consisted of 45 (48\%) patients. Thirty-eight patients (41\%) were classified as ERs, 20 (22\%) as LRs, and 35 (38\%) as NRs. On a multivariate regression analysis, the $\mathrm{P}$ group [odds ratio (OR), 3.24; $95 \%$ confidence interval (CI), 1.0810.45; $\mathrm{p}=0.036]$ and $\mathrm{NR}(\mathrm{OR}, 4.04 ; 95 \% \mathrm{CI}, 1.29-14.27 ; \mathrm{p}=0.016)$ were independent predictors of a poor outcome. ER $(47 \%, \mathrm{p}=0.01)$ and LR $(45 \%, \mathrm{p}=0.01)$ patients showed fewer poor outcomes than NR $(77 \%)$ patients, but the rate did not differ significantly between the ER and LR patients.

Conclusion Early mild clinical recovery did not predict a good outcome. The occlusion site was a stronger predictor of clinical improvement after IVtPA administration.
\end{abstract}

Key words: ischemic stroke, tissue plasminogen activator, outcome, occlusion site

(Intern Med 54: 3139-3144, 2015)

(DOI: 10.2169/internalmedicine.54.4990)

\section{Introduction}

Intravenous tissue plasminogen activator (IVtPA) is an effective treatment for patients with acute cerebral infarction. The treatment has been prescribed since $1995(1,2)$. In the approximately 20 years since, various reports have emerged describing IVtPA use. Several factors, including the initial stroke severity (3), patient age (4), pretreatment blood pressure (5), atrial fibrillation (6), and blood glucose concentration at admission (7), are reported to predict clinical outcomes.

In addition to these factors, the occlusion site before IVtPA and early dramatic recovery are reportedly associated with the clinical outcome. Saqqur et al. reported that a proximal major arterial occlusion decreased the rate of a

\footnotetext{
${ }^{1}$ Department of Neurological Science, Nippon Medical School, Japan, ${ }^{2}$ Department of Stroke, Institute of Brain and Blood Vessels, Mihara Memorial Hospital, Japan, ${ }^{3}$ Department of Neurology, Institute of Brain and Blood Vessels, Mihara Memorial Hospital, Japan and ${ }^{4}$ Department of Neurosurgery, Institute of Brain and Blood Vessels, Mihara Memorial Hospital, Japan Received for publication January 19, 2015; Accepted for publication April 16, 2015 Correspondence to Dr. Kentaro Suzuki, kentarow@nms.ac.jp
} 
good clinical outcome after IVtPA administration (8). Yeo et al. showed that an early dramatic recovery, defined as a reduction in the National Institutes of Health Stroke Scale (NIHSS) score of $\geq 10$ or a $0-4$ score at 2 hours and 24 hours after IVtPA administration, was an important factor in producing a good outcome (9). However, the relationship between a mild clinical improvement, which occurs more frequently than a dramatic recovery, and the clinical outcome (especially in patients with a major arterial occlusion), has not been investigated. A clear understanding of this relationship may enable the easier identification of candidates for additional treatment regimens (e.g., combined IVtPA and endovascular therapy).

In the present report, we therefore examined whether clinical improvement, defined as a reduction in the NIHSS score of $\geq 4$ or an NIHSS score of 0 at 2 or 24 hours after IVtPA treatment, was correlated with the clinical outcome at 3 months in patients with a major arterial occlusion in the anterior circulation.

\section{Materials and Methods}

\section{Study population and patient characteristics}

We retrospectively analyzed the medical records of the cerebral infarction patients who were treated at Mihara Memorial Hospital between November 2005 and October 2012. A total 2,184 cerebral infarction patients were admitted to our hospital over the 7-year study period. Consecutive patients with acute cerebral infarction in the anterior circulation, diagnosed by magnetic resonance imaging (MRI), and who received IVtPA without endovascular therapy within 3 hours of the onset of symptoms were enrolled. Of the total case population, 144 patients received IVtPA treatment without endovascular therapy. However, 49 patients were excluded from the analysis because the infarction was located in the posterior circulation, or because the occlusion site was not identified, and two patients were excluded because IVtPA treatment was initiated more than 3 hours after the onset of symptoms. Thus, 93 patients were enrolled in the present study. IVtPA with alteplase $(0.6 \mathrm{mg} / \mathrm{kg})$ was prescribed as described in the Japan Alteplase Clinical Trial (10). The following data were analyzed in all patients: age; gender; arterial systolic blood pressure at admission; serum low-density lipoprotein cholesterol (LDL) concentration; estimated glomerular filtration rate (eGFR); fasting blood glucose concentration at admission or the morning after admission; presence of atrial fibrillation; the duration to the start of IVtPA infusion; NIHSS score at admission, 2 hours, and 24 hours after IVtPA (11); and the site of arterial occlusion.

MRI studies, including diffusion-weighted imaging (DWI) and time-of-flight MR angiography (MRA), were performed on admission using a commercially available echo planar instrument operating at 3.0 T (GE Sigma EXCITE 3.0T HD, General Electric, Fairfield, USA). All baseline MRA data were evaluated at our institution by two experienced neurologists. The M1 region of the middle cerebral artery (MCA) included the sphenoidal segment, which lies posterior and parallel to the sphenoidal ridge. A proximal M1 occlusion was defined as an occlusion within $5 \mathrm{~mm}$ of the internal carotid artery (ICA) bifurcation, and a distal M1 occlusion was defined as occlusion beyond $5 \mathrm{~mm}$, as defined previously (12). The MCA distal region consisted of the superior and inferior trunks beginning at their division from M1. The diffusion-weighted imaging Alberta Stroke Program Early Computed Tomography Score (DWI-ASPECTS) was also assessed on admission (13). At 3 months after IVtPA, the functional outcome was assessed according to the modified Rankin Scale (mRS) score (14). An mRS score $<2$ was considered to indicate a good outcome, and a mRS score $\geq 2$ was considered to indicate a poor outcome.

\section{Neurologic status after IVtPA}

We assessed the neurological status at 2 hours and 24 hours after IVtPA. Early responders (ERs) were defined as patients in whom the NIHSS score decreased $\geq 4$ points or who had a NIHSS score of 0 within 2 hours of starting IVtPA therapy, as defined previously (15). Late responders (LRs) were defined as patients who required a period of 2 to 24 hours until the NIHSS score decreased $\geq 4$ points or who reached a score of 0 . Non-responders (NR) were defined as patients who had no change, an increase, or a $\geq 4$ point decrease in the NIHSS score more than 24 hours after the initiation of IVtPA therapy.

\section{Study process and methods}

The patients were classified into two groups according to the occlusion site. Patients with occlusion in the ICA or proximal M1 were placed in the $\mathrm{P}$ group, and those with occlusion in the distal M1 or distal MCA were placed in the D group. Patients with an ICA or proximal MCA occlusion were included in the $\mathrm{P}$ group regardless of the presence of anterior cerebral artery occlusion or multiple cerebral infarctions. The clinical characteristics, imaging findings, and the rates of ER, LR, and NR were compared between the P and D groups. The baseline characteristics of the study population were analyzed according to the clinical outcome at 3 months. Variables with a $\mathrm{p}$ value $<0.1$ in the univariate analysis were subjected to a multivariate analysis. A multiple logistic regression analysis was used to investigate the independent factors correlated with a poor outcome at 3 months. The cutoff NIHSS score at admission and the time to IVtPA administration differentiating the clinical outcome were calculated using a receiver operating characteristic (ROC) curve. The relative risk of a poor outcome was expressed as an odds ratio (OR) with a $95 \%$ confidence interval (CI). The correlation between the response type and clinical outcome at 3 months was analyzed to evaluate the relationship between the timing of a mild clinical improvement and the clinical outcome in patients with a major arterial occlusion. 


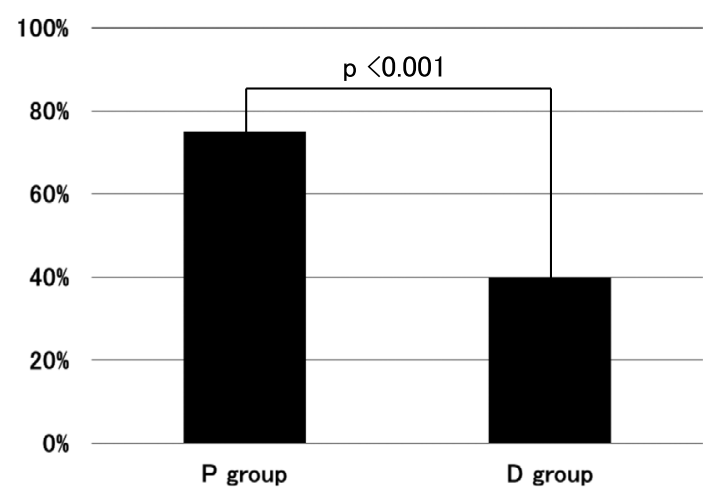

Figure 1. The rate of a poor clinical outcome at 3 months according to the occlusion site. A poor outcome was defined as a modified Rankin Scale score of $\geq 2$. The $P$ group comprised patients with an occlusion in the internal carotid artery (ICA) or a middle cerebral artery (MCA) M1 occlusion $<5 \mathbf{~ m m}$ from the ICA bifurcation. The D group comprised patients with occlusions $\geq 5 \mathrm{~mm}$ from the ICA bifurcation.

\section{Statistical analysis}

Statistical analysis included descriptive statistics [mean \pm standard deviation (SD), median with quartiles, or number (percentage) for categorical variables]. The $\chi^{2}$ and Fisher exact tests were used to compare proportions, and the twosample Student $t$ test, analyses of variance, and MannWhitney $U$-test were used to compare continuous response variables between the groups. A multiple regression analysis was performed using the P group, NR, ASPECTs, NIHSS score at admission $\geq 9$, time to IVtPA administration $\leq 118$ minutes, and eGFR. In all tests, $\mathrm{p}<0.05$ indicated statistical significance. The JMP 9 software program (SAS Institute, Cary, USA) was used to perform the statistical analyses.

\section{Results}

Ninety-three patients [median age, 74 (67-79) years; 54 (58\%) men; median NIHSS score, 11 (7-16)] were enrolled in the present study. Forty-eight (52\%) patients comprised the $\mathrm{P}$ group, and 45 (48\%) comprised the D group. Thirtyeight (41\%) of the patients were ERs, 20 (22\%) were LRs, and 35 (38\%) were NRs.

At 3 months, 40 (43\%) patients had a good outcome, and $53(57 \%)$ patients had a poor outcome. Thirty-five patients (73\%) in the $\mathrm{P}$ group and 18 patients $(40 \%)$ in the $\mathrm{D}$ group had a poor outcome $(\mathrm{p}<0.001$; Fig. 1$)$. A univariate regression analysis revealed that a low serum eGFR $(\mathrm{p}=0.04)$, a low ASPECTS ( $\mathrm{p}=0.01$ ), a high NIHSS score at admission $(\mathrm{p}=0.001)$, a short duration from the onset of symptoms to IVtPA administration $(\mathrm{p}=0.04)$, the presence of NR $(\mathrm{p}=$ $0.003)$, and being in the $P$ group $(p=0.001)$ were significantly associated with a poor outcome at 3 months (Table 1).

Table 2 summarizes the patient characteristics according to the occlusion site. Among the 93 patients, the ASPECTS was significantly lower $(\mathrm{p}=0.001)$, and the NIHSS score at admission $(\mathrm{p}<0.001)$, the rate of a poor outcome at 3 months $(\mathrm{p}<0.001)$, and the rate of NR patients were significantly higher $(\mathrm{p}=0.03)$ in the $\mathrm{P}$ group than in the $\mathrm{D}$ group. The numbers of cases according to the timing of improvement in the $\mathrm{P}$ and $\mathrm{D}$ groups were $17 / 48(35 \%)$ and $21 / 45$ (47\%) for the ER patients $(\mathrm{p}=0.27), 8 / 48(17 \%)$ and $12 / 45$ (27\%) for the LR patients $(\mathrm{p}=0.24)$, and $23 / 48(48 \%)$ and $12 / 45(27 \%)$ for the NR patients, respectively $(\mathrm{p}=0.03)$. There were no significant differences in any other of the baseline patient characteristics.

The cutoff NIHSS score at admission which predicted a poor outcome was $\geq 9$ on the ROC analysis, with a sensitivity of $64 \%$, a specificity of $82 \%$, a positive predictive value of $75 \%$, and a negative predictive value of $74 \%$. The cutoff duration between the onset of symptoms and IVtPA administration predicting a poor outcome was $\leq 118$ minutes on the ROC analysis, with a sensitivity of $64 \%$, a specificity of $82 \%$, a positive predictive value of $75 \%$, and a negative predictive value of $74 \%$. The multivariate regression analysis revealed that the P group (OR, 3.24; 95\% CI, 1.08-10.45; $\mathrm{p}=0.036$ ) was an independent predictor for a poor outcome, as were NR patient status (OR, 4.04; 95\% CI, 1.29-14.27; $\mathrm{p}=0.016)$, an NIHSS score at admission of $\geq 9$ (OR, 5.57; 95\% CI, 1.65-21.72; $\mathrm{p}=0.005$ ), and an onset to IVtPA duration of $\leq 118$ minutes $(\mathrm{OR}, 5.56$; 95\% CI, 1.65-21.72; $\mathrm{p}=$ 0.002) (Table 3).

Finally, we compared the effect of early and late response on the clinical outcome at 3 months. Both ERs and LRs were associated with fewer poor outcomes than NRs (ER vs. NR, $47 \%$, vs. $77 \%, p=0.01$; and LR vs. NR, $45 \%$ vs. $77 \%$, $\mathrm{p}=0.01)$. In the $\mathrm{P}$ group, $11 / 17(65 \%)$ patients had a poor outcome, and 4/8 (50\%) patients each in the ER and LR subpopulations had a poor outcome $(\mathrm{p}=0.48)$. All of the 23 NRs showed a poor outcome at 3 months. In the D group, patients with poor outcomes comprised 6/21 (29\%) and 4/12 (33\%) of the ER and LR subpopulations, respectively ( $\mathrm{p}=$ 0.78). Among the NRs of the D group, $7 / 12$ (58\%) showed a poor outcome at 3 months. The rate of a poor outcome did not differ significantly between the ER and LR subpopulations (Fig. 2).

\section{Discussion}

The occlusion site was an independent prognostic factor in our study. Patients in the P group were significantly more likely to have a poor clinical outcome. This finding was consistent with previous studies. In general, patients with an ICA occlusion show particularly low rates of recanalization and more frequently experience poor outcomes (16-19). Other studies have shown that IVtPA administration is less effective for proximal than for distal MCA occlusions. In particular, patients with a proximal MCA occlusion within 5 $\mathrm{mm}$ of the ICA bifurcation had significantly worse outcomes than those with distal MCA occlusion (12). Saqqur et al. retrospectively evaluated 335 cases of acute ischemic 
Table 1. Baseline Patient Characteristics According to the Clinical Outcome at 3 Months.

\begin{tabular}{llll}
\hline & $\begin{array}{l}\text { Good outcome } \\
\text { group } \\
\mathrm{n}=40\end{array}$ & $\begin{array}{l}\text { Poor outcome } \\
\text { group } \\
\mathrm{n}=53\end{array}$ & $\mathrm{p}$ \\
\hline Age, y & $70 \pm 11$ & $73 \pm 11$ & 0.19 \\
Male gender, no. (\%) & $25(63)$ & $29(55)$ & 0.5 \\
Systolic blood pressure, mm Hg & $153 \pm 28$ & $148 \pm 23$ & 0.29 \\
Diastolic blood pressure, mm Hg & $73 \pm 16$ & $80 \pm 13$ & 0.34 \\
LDL, mg/dL & $127 \pm 33$ & $127 \pm 27$ & 0.95 \\
eGFR, mg/min/1.73 m ${ }^{2}$ & $70 \pm 22$ & $61 \pm 21$ & 0.04 \\
Fasting blood sugar, mg/dL & $138 \pm 46$ & $148 \pm 51$ & 0.34 \\
Atrial fibrillation, no. (\%) & $15(38)$ & $26(49)$ & 0.29 \\
ASPECTS [interquartile range] & $9[7-10]$ & $8[7-10]$ & 0.01 \\
NIHSS score at admission [interquartile range] & $9[6-15]$ & $15[10-18]$ & $<0.001$ \\
Time to admin. of IVtPA, min & $134 \pm 27$ & $122 \pm 28$ & 0.04 \\
ER, no. (\%) & $20(50)$ & $18(34)$ & 0.1 \\
LR, no. (\%) & $11(28)$ & $9(17)$ & 0.13 \\
NR, no. (\%) & $8(20)$ & $27(51)$ & 0.003 \\
P group, no. (\%) & $13(33)$ & $35(66)$ & 0.001 \\
\hline LDL: low-density lipoprotein cholesterol, & eGFR: estimated & glomerular filtration rate, \\
ASPECTS: Alberta Stroke Program Early Computed Tomography Score, NIHSS: National \\
Institutes of Health Stroke Scale, mRS: modified Rankin Scale, tPA: tissue plasminogen activator, \\
ER: early responder, LR: late responder, NR: non-responder, P group: patients with occlusion in \\
the internal carotid artery (ICA) or with occlusion in the M1 area of the middle cerebral artery \\
(MCA) <5 mm from the ICA bifurcation & & \\
& & &
\end{tabular}

Table 2. Baseline Patient Characteristics According to Occlusion Site.

\begin{tabular}{llll}
\hline & $\begin{array}{l}\text { P group } \\
\mathrm{n}=48\end{array}$ & $\begin{array}{l}\text { D group } \\
\mathrm{n}=45\end{array}$ & $\mathrm{p}$ \\
\hline Age, y & $72 \pm 13$ & $72 \pm 9$ & 0.96 \\
Male gender, no. (\%) & $26(54)$ & $28(62)$ & 0.43 \\
Systolic blood pressure, mm Hg & $151 \pm 25$ & $149 \pm 22$ & 0.71 \\
Diastolic blood pressure, mm Hg & $80 \pm 17$ & $83 \pm 12$ & 0.31 \\
LDL, mg/dL & $129 \pm 33$ & $125 \pm 26$ & 0.93 \\
eGFR, mg/min/1.73 m ${ }^{2}$ & $66 \pm 22$ & $63 \pm 22$ & 0.55 \\
Fasting blood sugar, mg/dL & $148 \pm 52$ & $138 \pm 45$ & 0.32 \\
Atrial fibrillation, no. (\%) & $21(44)$ & $20(44)$ & 0.95 \\
ASPECTS [interquartile range] & $7[6-10]$ & $9[8-10]$ & 0.001 \\
NIHSS score at admission [interquartile range] & $15[10-18]$ & $9[6-15]$ & $<0.001$ \\
NIHSS score at admission 0-7, no. (\%) & $7(15)$ & $19(42)$ & \\
\multicolumn{1}{c}{ 8-15, no. (\%) } & $22(46)$ & $17(38)$ & \\
\multicolumn{1}{c}{ 16-, no. (\%) } & $19(40)$ & $9(20)$ & \\
Poor outcome at 3 months, no. (\%) & $35(73)$ & $18(40)$ & $<0.001$ \\
Time to admin. of IVtPA, min & $128 \pm 31$ & $127 \pm 25$ & 0.76 \\
ER, no. (\%) & $17(35)$ & $21(47)$ & 0.27 \\
LR, no. (\%) & $8(17)$ & $12(27)$ & 0.24 \\
NR, no. (\%) & $23(48)$ & $12(27)$ & 0.03 \\
\hline
\end{tabular}

P group: patients with occlusion in the internal carotid artery (ICA) or occlusion in the M1 area of the middle cerebral artery $<5 \mathrm{~mm}$ from the ICA bifurcation, D group: patients with occlusion $\geq 5 \mathrm{~mm}$ from the ICA bifurcation, LDL: low-density lipoprotein cholesterol, eGFR: estimated glomerular filtration rate, ASPECTS: Alberta Stroke Program Early Computed Tomography Score, NIHSS: National Institutes of Health Stroke Scale, mRS: modified Rankin Scale, tPA: tissue plasminogen activator, ER: early responder, LR: late responder, NR: non-responder

Table 3. Multivariate Logistic Regression Analysis for Independent Factors Associated with Poor Clinical Outcome.

\begin{tabular}{llll}
\hline & OR & $95 \%$ CI & $\mathrm{p}$ \\
\hline P group & 3.24 & $1.08-10.45$ & 0.036 \\
NR & 4.05 & $1.29-14.27$ & 0.016 \\
ASPECTS & 0.92 & $0.64-1.31$ & 0.641 \\
NIHSS score at admission $\geq 9$ & 5.57 & $1.65-21.72$ & 0.005 \\
Time to admin. of IVtPA $\leq 118$ & 5.56 & $1.82-19.92$ & 0.002 \\
eGFR & 0.98 & $0.96-1.01$ & 0.131 \\
\hline
\end{tabular}

OR: odds ratio, CI: confidence interval, eGFR: estimated glomerular filtration rate, ASPECTS: Alberta Stroke Program Early Computed Tomography Score, NIHSS: National Institutes of Health Stroke Scale, NR: non-responder stroke treated with IVtPA by analyzing the reperfusion rate and prognosis according to the occlusion site. They found that a good outcome was achieved in 90 of 260 patients (35\%) overall. In their population, good outcomes occurred according to the occlusion site as follows: distal middle cerebral artery, 52\% (50 of 96); proximal middle cerebral artery, $25 \%$ (33 of 131); tandem cervical internal carotid artery/middle cerebral artery, $21 \%$ (3 of 14); terminal internal cerebral artery, $18 \%$ ( 2 of 11 ); and basilar artery, $25 \%$ (2 of 8) (8).

We found that patients experiencing a clinical improvement within 24 hours after IVtPA administration were more 
P group

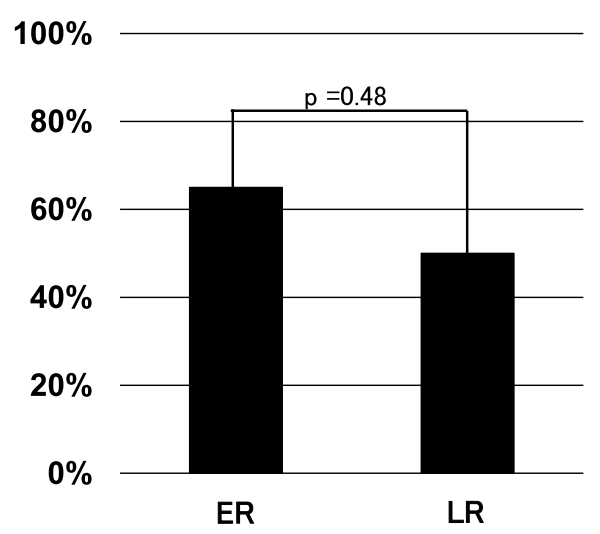

D group

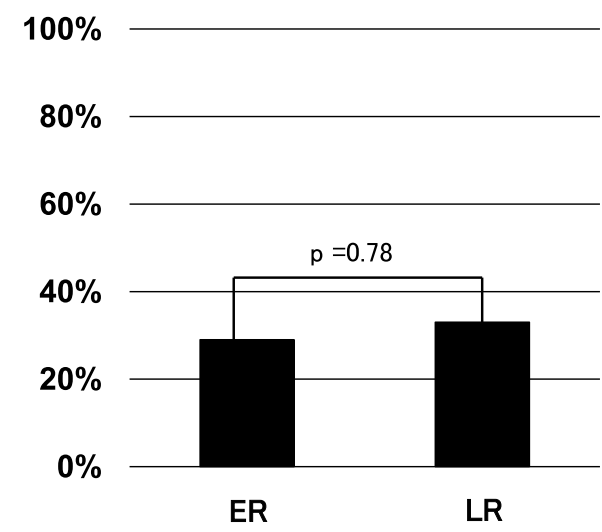

Figure 2. The association between a poor clinical outcome at 3 months and the occurrence of neurological improvement. A poor outcome was defined as a modified Rankin Scale score $<\mathbf{2}$. The clinical response was categorized as follows: early responder (ER), $\geq 4$-point improvement in the NIHSS score within $2 \mathrm{~h}$ after administering intravenous tissue plasminogen activator (IVtPA); and late responder (LR), 24-point improvement in the NIHSS score between 2 and $24 \mathrm{~h}$ after IVtPA administration.

likely to have good outcomes at 3 months after therapy than non-responding patients. However, the 3-month outcome did not differ between ERs and LRs after IVtPA administration. In addition, both ER and LR patients had a poor prognosis if they were in the $\mathrm{P}$ group. It is difficult to predict longterm outcomes based on mild initial clinical improvement a mere 2 hours after IVtPA. We therefore propose that the treatment method be determined according to the occlusion site rather than the speed of clinical improvement after IVtPA. We found that most patients with distal MCA occlusion achieved good outcomes after IVtPA treatment alone. However, most patients with ICA or proximal MCA occlusions did not achieve a good outcome. Abilleira et al. (20) analyzed the clinical outcomes in 536 patients with acute ischemic stroke, 285 of whom received IVtPA, and observed a sustained relationship between the onset of symptoms to endovascular therapy duration and a good outcome, regardless of the baseline NIHSS score. For patients with ICA or proximal MCA obstruction, we suggest shifting to a combined therapy without waiting to assess the clinical improvement.

Although most NR patients experienced a poor outcome, eight patients $(23 \%)$ had a good outcome. These eight patients had a significantly lower NIHSS score at admission [7 (5-16)] compared with the 27 patients who had a poor outcome [15 (8-16), $\mathrm{p}=0.08]$. This discrepancy may indicate that the eight patients had good collateral circulation, although this was not assessed. Eighteen patients (47\%) in the ER subgroup had a poor outcome. They showed a significantly higher NIHSS score at admission [15 (11-20)] than the 20 patients with a good outcome [9 (7-15); $\mathrm{p}=0.002]$. One reason that patients with ER experience poor outcomes may be the occurrence of re-occlusion. An excessively large infarct size and severe state at admission may be other reasons.

\section{Limitations}

This study is associated with some limitations. In Japan, alteplase is approved for administration at $0.6 \mathrm{mg} / \mathrm{kg}$, which differs from the approved dosage of $0.9 \mathrm{mg} / \mathrm{kg}$ in both the United States and internationally and this may have affected the clinical results following IVtPA administration. Second, this study was conducted at a single center using a small population. Third, patient eligibility for IVtPA therapy was determined on a case-by-case basis; the determination was based on the Japanese guidelines, which recommends against IVtPA therapy in patients with acute ischemic stroke if a high intensity lesion on DWI encompasses more than one-third of the MCA. These guidelines promote data bias during patient selection for IVtPA treatment. Notably, patients in whom IVtPA administration was relatively delayed showed better clinical outcomes, which likely reflects that severely affected patients were admitted to the hospital sooner than those with a low NIHSS score. Finally, we did not confirm the recanalization of the occluded vessel after IVtPA treatment. Therefore, we do not know whether the occluded vessel was a true predictor for the clinical outcome rather than recanalization.

\section{Conclusion}

The clinical improvement in the NIHSS score of $\geq 4$ points at 2 or 24 hours after IVtPA treatment did not predict a good outcome at 3 months. Rather, the site of vascular occlusion closely correlated with the clinical outcome at 3 months after an acute ischemic stroke.

The authors state that they have no Conflict of Interest (COI). 


\section{References}

1. The National Institute of Neurological Disorders and Stroke RTPA Stroke Study Group. Tissue plasminogen activator for acute ischemic stroke. N Engl J Med 333: 1581-1587, 1995.

2. Hacke W, Kaste M, Bluhmki E, et al; ECASS Investigators. Thrombolysis with alteplase 3 to 4.5 hours after acute ischemic stroke. N Engl J Med 359: 1317-1329, 2008.

3. Smith EE, Shobha N, Dai D, et al. Risk score for in-hospital ischemic stroke mortality derived and validated within the Get with the Guidelines stroke program. Circulation 122: 1496-1504, 2010.

4. Brown DL, Johnston KC, Wagner DP, Haley EC Jr. Predicting major neurological improvement with intravenous recombinant tissue plasminogen activator treatment of stroke. Stroke 35: 147-150, 2004.

5. Tsivgoulis G, Saqqur M, Sharma VK, Lao AY, Hill MD, Alexandrov AV. Association of pretreatment blood pressure with tissue plasminogen activator-induced arterial recanalization in acute ischemic stroke. Stroke 38: 961-966, 2007.

6. Saposnik G, Gladstone D, Raptis R, Zhou L, Hart RG. Atrial fibrillation in ischemic stroke: predicting response to thrombolysis and clinical outcomes. Stroke 44: 99-104, 2013.

7. Saposnik G, Di Legge S, Webster F, Hachinski V. Predictors of major neurologic improvement after thrombolysis in acute stroke. Neurology 65: 1169-1174, 2005.

8. Saqqur M, Uchino K, Demchuk AM, et al; CLOTBUST Investigators. Site of arterial occlusion identified by transcranial Doppler predicts the response to intravenous thrombolysis for stroke. Stroke 38: 948-954, 2007.

9. Yeo LL, Paliwal P, Teoh HL, et al. Early and continuous neurologic improvements after intravenous thrombolysis are strong predictors of favorable long-term outcomes in acute ischemic stroke. J Stroke Cerebrovasc Dis 22: 590-596, 2013.

10. Yamaguchi T, Mori E, Minematsu K, et al; Japan Alteplase Clinical Trial (J-ACT) Group. Alteplase at $0.6 \mathrm{mg} / \mathrm{kg}$ for acute ischemic stroke within 3 hours of onset: Japan Alteplase Clinical Trial (J-ACT). Stroke 37: 1810-1815, 2006.
11. Brott $\mathrm{T}$, Adams HP Jr, Olinger $\mathrm{CP}$, et al. Measurements of acute cerebral infarction: a clinical examination scale. Stroke 20: 864870, 1989.

12. Hirano T, Sasaki M, Mori E, Minematsu K, Nakagawara J, Yamaguchi T; Japan Alteplase Clinical Trial II Group. Residual vessel length on magnetic resonance angiography identifies poor responders to alteplase in acute middle cerebral artery occlusion patients: exploratory analysis of the Japan Alteplase Clinical Trial II. Stroke 41: 2828-2833, 2010.

13. Barber PA, Hill MD, Eliasziw M, et al; ASPECTS Study Group. Imaging of the brain in acute ischaemic stroke: comparison of computed tomography and magnetic resonance diffusion-weighted imaging. J Neurol Neurosurg Psychiatr 76: 1528-1533, 2005.

14. van Swieten JC, Koudstaal PJ, Visser MC, Schouten HJ, van Gijn J. Interobserver agreement for the assessment of handicap in stroke patients. Stroke 19: 604-607, 1988.

15. Kablau M, Kreisel SH, Sauer T, Binder J, Szabo K, Hennerici MG. Predictors and early outcome of hemorrhagic transformation after acute ischemic stroke. Cerebrovasc Dis 32: 334-341, 2011.

16. del Zoppo GJ, Poeck K, Pessin MS, et al. Recombinant tissue plasminogen activator in acute thrombotic and embolic stroke. Ann Neurol 32: 78-86, 1992.

17. Jansen $O$, von Kummer R, Forsting M, Hacke W, Sartor K. Thrombolytic therapy in acute occlusion of the intracranial internal carotid artery bifurcation. AJNR Am J Neuroradiol 16: 19771986, 1995.

18. Lee KY, Han SW, Kim SH, et al. Early recanalization after intravenous administration of recombinant tissue plasminogen activator as assessed by pre- and post-thrombolytic angiography in acute ischemic stroke patients. Stroke 38: 192-193, 2007.

19. Linfante I, Llinas RH, Selim M, et al. Clinical and vascular outcome in internal carotid artery versus middle cerebral artery occlusions after intravenous tissue plasminogen activator. Stroke 33: 2066-2071, 2002.

20. Abilleira S, Cardona P, Ribó M, et al; Catalan Stroke Code and Reperfusion Consortium. Outcomes of a contemporary cohort of 536 consecutive patients with acute ischemic stroke treated with endovascular therapy. Stroke 45: 1046-1052, 2014.

(C) 2015 The Japanese Society of Internal Medicine http://www.naika.or.jp/imonline/index.html 